\title{
Parasagittal Dural Space and Cerebrospinal Fluid (CSF) Changes Across the Lifespan in Healthy Adults: Implications for Glymphatic Flow
}

\author{
Kilian HETT ( $\square$ kilianhett@hotmail.com ) \\ Colin D. Mcknight \\ Vanderbilt-Ingram Cancer Center \\ Jarrod J. Eisma \\ Vanderbilt University Medical Center \\ Jason Elenberger \\ Vanderbilt University Medical Center \\ Jennifer D. Lindsey \\ Vanderbilt University Medical Center \\ Ciaran M. Considine \\ Vanderbilt University Medical Center \\ Daniel O. Claassen \\ Vanderbilt University Medical Center \\ Manus J. Donahue \\ Vanderbilt University Medical Center
}

Vanderbilt University Medical Center https://orcid.org/0000-0001-8831-4247

\section{Research Article}

Keywords: Parasagittal dura space, cerebral aqueduct, glymphatic, cerebrospinal fluid, aging

Posted Date: November 30th, 2021

DOI: https://doi.org/10.21203/rs.3.rs-1083306/v1

License: (c) (i) This work is licensed under a Creative Commons Attribution 4.0 International License.

Read Full License 


\section{Abstract}

Background: Recent studies have suggested the importance of a glymphatic clearance pathway for brain parenchymal metabolic waste products. One fundamental but relatively under-explored component of this pathway is the anatomic region surrounding the superior sagittal sinus, which has been hypothesized to encompass lymphatic vessels. This so-called parasagittal dural (PSD) space likely plays a physiologically significant role at the distal intracranial component of the human glymphatic circuit, yet owing to the relative novelty of this discovery, fundamental gaps persist in our knowledge of how this space changes with normal aging and intracranial bulk fluid transport.

Methods: We tested the hypotheses that volumetric magnetic resonance imaging (MRI) measures of the PSD space (i) are directly related to cerebrospinal fluid (CSF) flow at the cerebral aqueduct, and (ii) increase with age. Healthy participants $(n=62$; age range $=20-83$ years) provided informed, written consent and multi-modal 3 Tesla MRI was performed including phase contrast assessment of the CSF flow through the aqueduct of Sylvius, $T_{1}$-weighted and $T_{2}$-weighted MRI for tissue volume and PSD assessment. Standard anatomical and cognitive testing were applied to confirm inclusion criteria. PSD volume was extracted using a recently validated neural networks algorithm. Non-parametric regression models were applied to evaluate how PSD volume related to tissue volume and age cross-sectionally, and separately how PSD volume related to CSF flux (significance criteria: two-sided $p<0.05$ ).

Results: A significant enlargement of PSD volume in relation to normal aging ( $p<0.001$, Spearman's$=0.6)$, CSF volume $(p<0.001$, Spearman's- $=0.6)$ and bulk CSF flux through the aqueduct of Sylvius (anterograde and retrograde, $p<0.001$ ) were observed. The elevation in PSD volume was not significantly related to changes in tissue volume $(p=0.11$ and $p=0.24$ for gray and white matter, respectively). Findings are consistent with PSD volume increasing with age and bulk CSF flux.

Conclusions: The findings of this study are two folds, first they highlight the feasibility of quantifying PSD volume non-invasively in vivo in humans using machine learning and non-contrast MRI. Second, that PSD volume increases with age, and relates to bulk CSF volume and flux. Values reported should provide useful normative ranges for how PSD volume adjusts with age, which will serve as a necessary prerequisite for comparisons to persons with neurodegenerative disorders.

\section{Introduction}

The proposed glial-lymphatic system, or glymphatic system, has prompted a reassessment of cerebrospinal fluid (CSF) as an important mediator in neurologic waste product clearance. Increasing animal and human evidence supports the presence of this highly organized system whereby fluid passes from the subarachnoid space to the periarterial space en route to the brain parenchymal interstitial space [1]-[4]. Net fluid motion is also observed exiting the interstitial space via the perivenous space, en route back to the subarachnoid space. Impairment of this system has been suggested as a mechanism of waste product accumulation which may underly the development of multiple neurodegenerative diseases 
with unknown etiology, including but not limited to Alzheimer's disease, Parkinson's disease, and multiple sclerosis [5], [6].

Classically, the central nervous system (CNS) is recognized to be devoid of lymphatic vessels. However, there is emerging evidence that lymphatic vessels are indeed present in the region surrounding the superior sagittal sinus [7]. Following intrathecal gadolinium contrast administration in 18 human subjects, contrast was noted to concentrate in the CSF spaces near the vertex. With passage of time, contrast progressively accumulated within the tissue surrounding the superior sagittal sinus, or the parasagittal dural (PSD) space [8]. These findings suggest that trans-arachnoid molecular passage occurs in this region and highlights the PSD space as a potential bridging link between the glymphatic circulation within the human brain and the dural lymphatic vessels. Despite this, there is limited knowledge of how variance in PSD space relates to aging, brain health, and intracranial CSF flow.

More specifically, the primary source of CSF production is the choroid plexus (ChP), which is estimated to produce CSF at a rate of approximately $20 \mathrm{ml} / \mathrm{h}$ in adult humans [9]. While most of the ChP tissue resides within the atria of the lateral ventricles, there is choroidal tissue throughout the ventricular system in rough proportion to the overall size of the ventricular components. CSF produced in the lateral and third ventricles traverses the cerebral aqueduct (e.g., aqueduct of Sylvius) en route to the 4th ventricle and on to the more diffuse subarachnoid space [10], [11]. As the cerebral aqueduct represents the sole pathway for CSF efflux from the lateral and third ventricles, measurement of flow in this region offers an opportunity to quantify fluid production by the third and lateral ventricles, which comprise the largest ChP complexes. To measure CSF efflux in the cerebral aqueduct, magnetic resonance (MR) phase contrast sequences, traditionally sensitive to arterial or venous flow, have been re-parameterized to enable quantitative CSF flow, primarily by pairing with cardiac phase and reducing the velocity encoding gradient to coincide with CSF flow (typically 10-20 cm/s). [12]. Aberrant phase contrast flow parameters have long been associated with idiopathic normal pressure hydrocephalus [13]. CSF flow through the cerebral aqueduct is also dependent on cardiac phase and has been shown to be highest in older adults and in males vs. females [14]. These findings are consistent with previous studies that have shown age, and sex dependencies of CSF on tissue volume [15], [16], and suggest that CSF flow profiles may relate to total CSF volume.

The logical extension of this work is to understand how such CSF flow parameters relate to quantitative estimates of the PSD volume. Here, we test the hypotheses that increases in PSD volume parallel changes in CSF flow through the cerebral aqueduct, and additionally that PSD volume increases with advancing age. Findings are interpreted in the context of the growing literature on glymphatic physiology and bulk CSF flow.

\section{Materials And Methods}

\section{Participants}


All participants provided informed, written consent in accordance with the local institutional review board (IRB) and consistent with the Declaration of Helsinki and its amendments. All participants were scanned between January 2020 and September 2021 at Vanderbilt University Medical Center. Inclusion criteria for healthy control participants: age=20-83 years, no history of cerebrovascular disease, anemia, psychosis, or neurological disorder including but not limited to prior overt stroke, sickle cell anemia, schizophrenia, bipolar disorder, Alzheimer's disease, Parkinson's disease, or multiple sclerosis. Presence of non-specific white matter lesions was not an exclusion criterion, as these lesions are extremely prevalent with aging, and we sought our cohort to be generalizable and representative. Clinical history was reviewed by a board-certified Neurologist (DOC; experience $=14$ years) and anatomical imaging and angiography by a board-certified neuroradiologist $(\mathrm{CDM}$; experience $=12$ years $)$ to ensure that inclusion criteria were met.

\section{Acquisition}

All participants were scanned at 3.0T (Philips Healthcare, Best, The Netherlands) using body coil radiofrequency transmission and SENSE array 32-channel reception. The scan protocol included standard anatomical imaging consisting of 3D $T_{1}$-weighted MPRAGE (echo time $=8.1 \mathrm{~ms}$, repetition time $=3.7 \mathrm{~ms}$, flip angle $=8^{\circ}$, resolution $=1 \times 1 \times 1 \mathrm{~mm}$ ), 3D $T_{2}$-weighted VISTA (echo time $=0.31 \mathrm{~ms}$, repetition time $=2.7 \mathrm{~ms}$, and spatial resolution $=0.78 \times 0.78 \times 0.78 \mathrm{~mm}$ ), 2D $T_{2}$-weighted FLAIR (echo time $=120 \mathrm{~ms}$, repetition time $=11,000 \mathrm{~ms}$, spatial resolution $=1 \times 1 \times 4 \mathrm{~mm}$ ), 3D time-of-flight magnetic resonance angiography (echo time $=3.45 \mathrm{~ms}$, repetition time $=23 \mathrm{~ms}$, spatial resolution $=0.39 \times 0.39 \times 1.4 \mathrm{~mm}$ ), and diffusion weighted imaging (DWI) (echo time $=83 \mathrm{~ms}$, repetition time $=2923$, b-value $=1000 \mathrm{~s} / \mathrm{mm}^{2}$; spatial resolution $=1.8 \times 1.8 \times 4 \mathrm{~mm}$ ). These scans were primarily used for confirming healthy status and ensuring inclusion criteria; $T_{1}$-weighted and $T_{2}$-weighted scans were additionally used for brain parenchymal and PSD volume segmentation as described below.

CSF movement was recorded within the aqueduct of Sylvius. Here, four MR-compatible ECG electrodes were placed to enable retrospective cardiac phase correction. A single slice orthogonal to the aqueduct of Sylvius was placed above the location of the 4th ventricle, where the aqueduct is bound by the tectum posteriorly and mid-brain anteriorly. A velocity encoding gradient of $12 \mathrm{~cm} / \mathrm{s}$ was applied along with 12 measurements over the cardiac cycle.

\section{Analysis}

CSF Flux: To estimate CSF flux in the aqueduct of Sylvius, we followed the acquisition protocol proposed in [12]. CSF flow parameters were quantified on the scanner console using the Q-Flow package provided by Philips Healthcare. The algorithm effectively subtracts phase contrast data acquired with the polarity of the bipolar phase contrast gradients reversed, which leads to computation of the following flow parameters: mean flux $(\mathrm{ml} / \mathrm{s})$, max anterograde flux $(\mathrm{ml} / \mathrm{s})$, max retrograde flux $(\mathrm{ml} / \mathrm{s})$.

Anatomical characteristics: All brain volumes were calculated using the $T_{1}$-weighted acquisitions. First, intracranial volume (ICV) was estimated using the brain mask computed by the advanced normalization 
tools (ANTs) package [17], which utilized the MNI ICBM-152 version as a template [18]. CSF, gray matter (GM), and white matter (WM) volumes were calculated using the Atropos method [19].

Parasagittal dural space quantification: PSD volumes were computed using a semi-supervised segmentation method based on a combination of a fully connected neural network (F-CNN) and voxel clustering based on a gaussian mixture model (GMM) to label voxels as PSD or sagittal sinus based on their $T_{2}$-weighted MRI signal intensities. The first step of our method used an F-CNN to extract parasagittal space; this deep-learning model was trained using $20 T_{2}$-weighted MRI scans, which provides the best contrast to the adjacent subarachnoid space. This deep-learning model aims to estimate a binary mask of parasagittal space which includes both the superior sagittal sinus and contributing veins in the region of the PSD and the parasagittal dural space. Once the parasagittal space mask was computed, voxels belonging to the parasagittal mask were labeled as PSD, or superior sagittal sinus/contributing veins.

To achieve this, bias field inhomogeneity was corrected using N4 inhomogeneity correction [20]. Next, in order to reduce anatomical variability, all $T_{2}$-weighted MRI images were aligned to the MNI template [18] using non-linear registration computed with ANTs [17] with a control spacing point set to $2 \mathrm{~mm}$. This value provides equipoise between the robustness of the registration (i.e., limitation of eventual registration artifacts) and increase of inter-subject similarity of the PSD. The PSD volumes are obtained using a semi-supervised machine learning method as described below.

First, a board-certified neuroradiologist (CDM; experience $=12$ years) manually segmented the parasagittal space of $20 T_{2}$-weighted scans. This region of interest contains the superior sagittal sinus venous volume and PSD space volume along the sinus. Next, manual segmentation maps were used to train an automatic segmentation method based on an F-CNN using a U-Net architecture [21]. U-Net architecture was chosen for its good performance with medical image segmentation task and its ability to deal with limited training data size. In total, 180 overlapping patches were used to reconstruct the parasagittal space. Finally, a GMM was fit, within the estimated parasagittal mask, using an expectationmaximization strategy on the $T_{2}$-weighted MRI.

Thus, the final maps provide labels for voxels belonging to the PSD space (hyperintense on the $T_{2^{-}}$ weighted scans) and venous structures including the superior sagittal sinus and contributing veins (hypointense in the $T_{2}$-weighted scans). Resulting segmentation maps were transformed to native space using the inverse transform. Therefore, all subsequent analyses are performed in the native space of the $T_{2}$-weighted scans. All segmentation maps (PSD and tissue masks) were validated by a neuroradiologist (CDM; experience $=12$ years).

\section{Statistical analysis}

To assess the validity of our hypotheses, we used a generalized linear model (GLM). We defined three multivariate models to assess three different hypotheses. 
1. Demographics and intracranial cavity volume (ICV): we defined a model with PSD and max absolute flux (i.e., anteretrograde and retrograde) as separate dependent variables and age, sex, and intracranial volume as independent variables.

2. Demographics and tissue volumes (GM, WM, and CSF): we defined a model with PSD and max CSF flux as separate dependent variables and age, sex, CSF, GM, and WM volumes as independent variables.

3. PSD space and bulk CSF Flux: we defined a model with PSD volume as the dependent variable and max CSF flux, age, and sex as independent variables.

In addition of these linear models, we assessed the correlation of each pair of features using Spearman's rank correlation coefficient. This was tested using analysis of variance (ANOVA) and corrected for multiple comparison using false discovery rate (FDR) [22]. All reported $p$-values are reported as raw and corrected with FDR with significance fixed to 0.05. All analyses were performed in Matlab (Mathworks) using the statistical toolbox.

\section{Results}

\section{Demographics}

A summary of participant demographics is provided in Table 1. In total, 62 participants were included, with an age ranging from 20 to 83 years inclusive. All participants met neurological and radiological inclusion criteria as defined in the Materials and Methods. 
Table 1

Demographic description of the cohort. Significance was evaluated using a Kruskal-Wallis test. ICV: intra-cranial volume; CSF: Cerebrospinal fluid; GM: Gray matter; WM: White matter; PSD: Parasagittal dura; Retrograde and anteretrograde designated the maximum flux in each direction (i.e., 3rd and lateral ventricles to 4th ventricles, and inversely).

\begin{tabular}{|llllll|}
\hline & Youth & Middle & Elderly & Total & P \\
& $20-39$ & $40-59$ & $60-83$ & $20-83$ & \\
\hline Age (year) & $27.5(5.6)$ & $51.3(6.5)$ & $70.2(5.9)$ & $50.4(18.0)$ & - \\
\hline Number of scans & 20 & 22 & 23 & 65 & - \\
\hline Sex $(F / M)$ & $15 / 5$ & $12 / 10$ & $15 / 8$ & $42 / 23$ & 0.3 \\
\hline ICV cm ${ }^{3}(\sigma)$ & $1340(101)$ & $1407(143)$ & $1360(135)$ & $1371(130)$ & 0.3 \\
\hline CSF cm ${ }^{3}(\sigma)$ & $239(31)$ & $279(50)$ & $318(44)$ & $280(53)$ & $<0.01$ \\
\hline GM cm ${ }^{3}(\sigma)$ & $691(63)$ & $690(63)$ & $645(55)$ & $675(59)$ & $<0.01$ \\
\hline WM cm ${ }^{3}(\sigma)$ & $409(22)$ & $438(55)$ & $396(54)$ & $415(52)$ & 0.06 \\
\hline Anterograde mL/s $(\sigma)$ & $0.08(0.03)$ & $0.08(0.04)$ & $0.13(0.07)$ & $0.10(0.06)$ & 0.04 \\
\hline Retrograde mL/s $(\sigma)$ & $-0.10(0.04)$ & $-0.11(0.05)$ & $-0.16(0.07)$ & $-0.12(0.06)$ & $<0.01$ \\
\hline PSD volume cm ${ }^{3}(\sigma)$ & $7.05(1.34)$ & $8.31(2.23)$ & $9.66(2.06)$ & $8.36(2.17)$ & $<0.01$ \\
\hline
\end{tabular}

\section{Volumetric results}

No significant difference in ICV over the different age groups of 20-39 years, 40-59 years, or 60-83 years (mean ICV equal to $1371 \mathrm{~cm}^{3}, \sigma=130 \mathrm{~cm}^{3}$ ) or sex was observed. The analysis of the relationship between brain tissue volume and age indicates that GM significantly declines with age with p-value $<0.001$ and $\rho$ $=0.79$, while no significant relationship was observed for WM using a linear model. Alternatively, CSF volumes significantly increased with age ( $\mathrm{p}$-value $<0.001$ and $\rho=0.79$; see Table 1 and Supplementary Table A1).

Figure 1 shows an example of the PSD segmentation process and Figure 2 demonstrates the quantification process for CSF flux. Figures 3-4 summarize the relationships between tissue volumes, including PSD volume, and age, whereas Figure 5 summarizes the relationship between PSD volume and CSF flux. PSD volume was found to have a significant relationship with age: $p<0.001$ ( $q$-values=0.009) and $\rho=0.59$. Like PSD, bulk CSF flux (i.e., anteretrograde and retrograde) were also found to have a significant relationship with age: $\mathrm{p}=0.010$, and $\mathrm{p}<0.001$ (q-values $=0.030$, and 0.002 ) and $\rho=0.36$, and -0.40 for PSD volume, anteretrograde, and retrograde flux, respectively. A significant sex relationship was 
observed with PSD volume ( $p$-value<0.001). However, sex difference was not significant in relation to bulk CSF flux. These findings are consistent with PSD volume being directly related to CSF flux through the aqueduct, as well as being elevated in males versus females.

The investigation of the relationship of PSD volume and CSF flux with ICV are detailed in Table A2 (see supplementary materials). We observed that ICV and PSD volume are not significantly related when sex is included as a covariate. Moreover, Spearman's correlation coefficient does not indicate any explanatory characteristic of ICV over the PSD volume ( $\rho=0.1$ and 0.2 , for male and female, respectively).

\section{Relationship with brain tissue volumes}

After confirming the correlation of both proposed glymphatic markers (i.e., bulk CSF flux and PSD volume) with age and sex, we analyzed their relationships with brain tissue volumes. We observed no significant evidence of a relationship between brain tissue volume and either anteretrograde or retrograde CSF flux. Maximum retrograde CSF flux showed a significant relationship with CSF volume before controlling for multiple hypothesis testing with a p-value of 0.04 , but this did not retain significance after FDR correction ( $q$-value $=0.08$ ). Spearman's rank correlation method indicated a correlation of retrograde CSF flux and CSF volume, with a correlation coefficient $\rho=-0.45$ ( $\mathrm{p}$-value $<0.001$ ). GM and WM volumes were not significantly related to anteretrograde or retrograde flux $(p=0.69,0.42$ and $0.14,0.08$ anteretrograde and retrograde, respectively) (Figures 4-5, Supplementary Table A3-A4).

Using the same method of relationship, we assessed the relationship between PSD volume and brain tissue and CSF volumes (Figures 3-4). No evidence of a correlation between PSD enlargement and GM or WM volume was observed. PSD volume was observed to positively correlate with CSF volume with $p$ values $=0.02$ ( $q$-value $=0.04$ ). In addition, the correlation analysis using Spearman's correlation coefficient indicated a strong correlation of PSD volume with a correlation coefficient $\rho=0.6(p<0.001)$.

\section{Parasagittal dura space and bulk CSF flux}

Two linear models were applied to evaluate the relationship between PSD volume and CSF flux in the cerebral aqueduct. The results provide evidence for a significant correlation of PSD volume and CSF flux for both anteretrograde and retrograde directionality. The model indicates that anteretrograde CSF flux correlates with PSD volume with a p-value equal to 0.01 (q-value equal to 0.04), and a Spearman's correlation coefficient of 0.36 ( $p$-value $=0.001)$. Retrograde CSF flux show stronger correlation, with a $p$ value $=0.001$ ( $q$-value $=0.004$ ), and a Spearman's correlation coefficient of -0.50 ( $p$-value inferior to 0.001 ). Figure 6 shows representative cases of a young and older adult which demonstrate the observation of PSD volume increasing with age.

\section{Discussion}

Emerging evidence supportive of a human glymphatic system and its underlying role in cerebral waste clearance has prompted a reevaluation of neuro-fluid circulation and relevance. It has recently been suggested that fluid clearance may occur along lymphatic channels, which co-localize with the anatomic 
region surrounding the superior sagittal sinus [7] and compelling evidence supports the presence of transarachnoid molecular passage in this region [23]. Our understanding of the anatomical and functional relevance of this region remains incomplete, partly due to a lack of robust methods for evaluating this space on neuroimaging, as well as how this space changes with age, sex, and standard measures of bulk CSF flow. Here, we provide evidence that the PSD volume increases with age, and also directly relates to CSF flux through the cerebral aqueduct.

As such, these findings suggest that the PSD is an important component of the distal end of the glymphatic system whereby fluid egresses from the intracranial compartment. We observed a significant enlargement of PSD space in relation to normal aging ( $p$-value $<0.001, \rho=0.6)$, CSF volume ( $p$-value < $0.001, \rho=0.6$ ) and CSF flux in the cerebral aqueduct (retrograde and anteretrograde, $\mathrm{p}$-values $<0.001, \rho$ $=0.32$ and -50 , respectively).

These findings should also be considered in the context of the growing literature on PSD anatomy. The initial work demonstrating trans-arachnoid molecular passage in the PSD region was conducted by introducing gadolinium contrast into the subarachnoid space via lumbar puncture [23], with subsequent $T_{1}$ weighted imaging detailing contrast progression to the PSD. A subsequent study assessed the "perisinus lymphatic space volume" retrospectively assessing $T_{1}$ post contrast imaging in patients suspected of having brain metastasis [24]. In this study we use submillimeter 3D $T_{2}$-weighted imaging of the brain to assess similar volumes. The technique we used allows for clear distinction of the PSD from both the adjacent subarachnoid space as well as the superior sagittal sinus and feeding cortical veins (see Figures 4-5). Our delineation of this PSD volume very closely matches the 2D appearance and the 3D volumetric maps as detailed by Ringstad et al [23]. Importantly, this method obviates the need for contrast enhanced imaging to determine the PSD volume. Accordingly, this method can likely be applied to numerous noncontract imaging datasets of various patient cohorts in the public domain. We anticipate that the noncontrast nature of our method will accelerate future study of the PSD, which will in turn further define the relevance of this region in the context of glymphatic flow and CNS CSF clearance.

Our results also confirm the previous findings by Park et al that demonstrated increasing PSD volumes with age (Figure 6). Our study expands upon this work to demonstrate that these increased PSD volumes are associated with increased CSF volume, though is not related to brain parenchymal volume. This observation is significant, as it implies that increased PSD cannot be explained simply by brain volume loss and therefore my provide insight into the pathophysiology of other neurodegenerative processes. Our study benefits from a wider cohort age range compared to this previous work (mean age $=62.1, \sigma=10.9$ years, compared to our cohort with mean age $=50.4, \sigma=18$ years). We believe that the current cohort which ranged in age from 20 to 83 years enables us to detect evolution of PSD in a more comprehensive manner over the approximate adult human lifespan.

\section{Cerebral aqueductal flux and parasagittal dural space volume}


PSD volumes were significantly correlated to maximum anteretrograde and retrograde CSF flux in the cerebral aqueduct. The cerebral aqueduct and PSD are at the proximal and distal ends of the glymphatic circuit respectively. This correlation presents further evidence of an organized system of CSF circulation and metabolism and suggests of complex physiologic interplay between various anatomic structures. Further study is needed across the human lifespan and in various disease cohorts to determine the sequence of dysfunction across the various regions of the glymphatic circuit. Investigations which characterize these findings across differing neurodegenerative disease cohorts may sheds light on how aberrant CSF flow contributes to various neurodegenerative conditions.

Finally, we also assessed for a relationship between CSF flux in the cerebral aqueduct with white and gray matter volumes, CSF volume, gender, and age. Our experiments indicate that CSF volumes show significant correlation with maximum retrograde CSF flux in the cerebral aqueduct. This again supports that these findings cannot be fully explained by brain volume loss.

In one prior study investigating the relationship of different CSF dynamics at the level of the cerebral aqueduct, CSF movement was shown to be dependent on age and gender [14]. Only part of these readouts can be confirmed by our experiments, even though we see correlation with age, sex difference was inconclusive in our analysis. However, it is noteworthy that maximum anteretrograde and retrograde CSF flux have not been directly investigated in this previous study, this could explain why we did not observe gender dependencies with CSF flux in our data.

The study findings should also be considered in light of several limitations. First, we evaluated the PSD volume across the life-span cross-sectionally as is common in neuroimaging studies, rather than longitudinally. Second, while the largest cohort of PSD volume data presented to date, the sample size of 62 was moderate and presented multiple co-variates from being included in analysis. However, we characterized the health of each participant both radiologically and neurologically as described in the inclusion criteria, and all participants met rigorous healthy volunteer criteria. Given the moderate sample size, we also focused hypotheses on those that could be tested responsibly with the sample size.

\section{Conclusions}

Findings highlight the feasibility of quantifying PSD volume non-invasively in vivo in humans using machine learning and MRI, that PSD volume increases with age, and that PSD volume relates to bulk CSF volume and flux. Values reported should provide useful normative ranges for how PSD volume adjusts with age, which will serve as a necessary pre-requisite for comparisons to persons with neurodegenerative disorders. Findings also motivate the evolving hypothesis that aging compromises the efficiency of glymphatic circulation, leading to PSD hypertrophy. These processes may contribute to agerelated neurological changes and possible vulnerability to further pathological disruption of CSF clearance processes.

\section{Declarations}




\section{Acknowledgement}

This study has been carried out with the financial support of NIH/NIA grant 5R01AG062574 and NIH/NCCIH grant 1R01AT011456. Vanderbilt University Medical Center Institutional Review Board has approved this study.

\section{Contributions}

$\mathrm{KH}, \mathrm{CDM}, \mathrm{CMC}, \mathrm{DOC}$, and MJD contributed to study design and interpretation of data. MJD, JE, and JJE contributed to data acquisitions. CDM contributed to the review of the data. $\mathrm{KH}$ and MJD contributed to analysis and development of new methods. KH, CDM, CMC, DOC, JE, JJE, JL and MJD contributed to writing the manuscript.

\section{Ethics approval and consent to participate}

All participants provided informed, written consent in accordance with the local institutional review board (IRB) and consistent with the Declaration of Helsinki and its amendments.

\section{Consent for publication}

Not applicable

\section{Competing interests}

No competing interests to declare

\section{Availability of data and materials}

The data that support the findings of this study are available from the corresponding author, MJD, upon reasonable request.

\section{References}

1. J. J. Iliff et al., "A paravascular pathway facilitates CSF flow through the brain parenchyma and the clearance of interstitial solutes, including amyloid $\beta$," Sci. Transl. Med., vol. 4, no. 147, Aug. 2012, doi: 10.1126/SCITRANSLMED.3003748.

2. L. Xie et al., "Sleep drives metabolite clearance from the adult brain,” Science (80-.)., vol. 342, no. 6156, pp. 373-377, 2013, doi: 10.1126/SCIENCE.1241224.

3. G. Ringstad et al., "Brain-wide glymphatic enhancement and clearance in humans assessed with MRI," JCI Insight, vol. 3, no. 13, Jul. 2018, doi: 10.1172/JCI.INSIGHT.121537.

4. G. Ringstad, S. A. S. Vatnehol, and P. K. Eide, "Glymphatic MRI in idiopathic normal pressure hydrocephalus," Brain, vol. 140, no. 10, pp. 2691-2705, Oct. 2017, doi: 10.1093/BRAIN/AWX191. 
5. T. Taoka et al., "Evaluation of glymphatic system activity with the diffusion MR technique: diffusion tensor image analysis along the perivascular space (DTI-ALPS) in Alzheimer's disease cases," Jpn J Radiol, vol. 35, pp. 172-178, 2017, doi: 10.1007/s11604-017-0617-z.

6. C. D. McKnight et al., "Diffusion along perivascular spaces reveals evidence supportive of glymphatic function impairment in Parkinson disease," Parkinsonism Relat. Disord., vol. 89, pp. 98-104, Aug. 2021, doi: 10.1016/J.PARKRELDIS.2021.06.004.

7. M. Absinta et al., "Human and nonhuman primate meninges harbor lymphatic vessels that can be visualized noninvasively by MRI," Elife, vol. 6, Oct. 2017, doi: 10.7554/ELIFE.29738.

8. G. Ringstad and P. K. Eide, "Cerebrospinal fluid tracer efflux to parasagittal dura in humans," Nat. Commun., vol. 11, no. 1, Dec. 2020, doi: 10.1038/s41467-019-14195-x.

9. John E. Hall and Michael E. Hall, Guyton and Hall Textbook of Medical Physiology, 14th ed..

10. H. Tumani, A. Huss, and F. Bachhuber, "The cerebrospinal fluid and barriers - anatomic and physiologic considerations," Handb. Clin. Neurol., vol. 146, pp. 21-32, Jan. 2018, doi: 10.1016/B9780-12-804279-3.00002-2.

11. L. Sakka, G. Coll, and J. Chazal, "Anatomy and physiology of cerebrospinal fluid," Eur. Ann. Otorhinolaryngol. Head Neck Dis., vol. 128, no. 6, pp. 309-316, Dec. 2011, doi: 10.1016/J.ANORL.2011.03.002.

12. A. Najafi, T. D. J. Sartoretti, C. A. Binkert, S. Sartoretti-Schefer, and M. Wyss, "CSF flow quantification in the cerebral aqueduct using phase contrast MR - How to do it properly," 2018, Accessed: Sep. 28, 2021. [Online]. Available: https://epos.myesr.org/poster/esr/ecr2018/C-1577/Personal information\#poster.

13. P. H. Luetmer et al., "Measurement of Cerebrospinal Fluid Flow at the Cerebral Aqueduct by Use of Phase-contrast Magnetic Resonance Imaging: Technique Validation and Utility in Diagnosing Idiopathic Normal Pressure Hydrocephalus," Neurosurgery, vol. 50, no. 3, pp. 534-543, Mar. 2002, doi: 10.1097/00006123-200203000-00020.

14. T. Sartoretti et al., "Sex and Age Dependencies of Aqueductal Cerebrospinal Fluid Dynamics Parameters in Healthy Subjects," Front. Aging Neurosci., vol. 0, no. JUL, p. 199, 2019, doi: 10.3389/FNAGI.2019.00199.

15. P. Coupé, G. Catheline, E. Lanuza, and J. V. Manjón, "Towards a unified analysis of brain maturation and aging across the entire lifespan: A MRI analysis," Hum. Brain Mapp., vol. 38, no. 11, pp. 55015518, 2017, doi: 10.1002/hbm.23743.

16. L. O. Wahlund et al., "The brain in healthy aged individuals: MR imaging.," https://doi.org/10.1148/radiology.174.3.2305048, vol. 174, no. 3 I, pp. 675-679, Mar. 1990, doi: 10.1148/RADIOLOGY.174.3.2305048.

17. B. B. Avants, N. J. Tustison, G. Song, P. A. Cook, A. Klein, and J. C. Gee, "A reproducible evaluation of ANTs similarity metric performance in brain image registration," Neuroimage, vol. 54, no. 3, pp. 2033-2044, Feb. 2011, doi: 10.1016/j.neuroimage.2010.09.025. 
18. V. Fonov, A. C. Evans, K. Botteron, C. R. Almli, R. C. McKinstry, and D. L. Collins, "Unbiased average age-appropriate atlases for pediatric studies," Neuroimage, vol. 54, no. 1, pp. 313-327, Jan. 2011, doi: 10.1016/j.neuroimage.2010.07.033.

19. B. B. Avants, N. J. Tustison, J. Wu, P. A. Cook, and J. C. Gee, "An Open Source Multivariate Framework for n-Tissue Segmentation with Evaluation on Public Data," Neuroinformatics 2011 94, vol. 9, no. 4, pp. 381-400, Mar. 2011, doi: 10.1007/S12021-011-9109-Y.

20. N. J. Tustison et al., "N4ITK: Improved N3 bias correction," IEEE Trans. Med. Imaging, vol. 29, no. 6, pp. 1310-1320, Jun. 2010, doi: 10.1109/TMI.2010.2046908.

21. O. Ronneberger, P. Fischer, and T. Brox, "U-net: Convolutional networks for biomedical image segmentation," Lect. Notes Comput. Sci. (including Subser. Lect. Notes Artif. Intell. Lect. Notes Bioinformatics), vol. 9351, pp. 234-241, 2015, doi: 10.1007/978-3-319-24574-4_28.

22. Y. Benjamini and Y. Hochberg, "Controlling the False Discovery Rate: A Practical and Powerful Approach to Multiple Testing," J. R. Stat. Soc. Ser. B, vol. 57, no. 1, pp. 289-300, Jan. 1995, doi: 10.1111/j.2517-6161.1995.tb02031.x.

23. G. Ringstad and P. K. Eide, "Cerebrospinal fluid tracer efflux to parasagittal dura in humans," Nat. Commun. 2020 111, vol. 11, no. 1, pp. 1-9, Jan. 2020, doi: 10.1038/s41467-019-14195-x.

24. M. Park, J. W. Kim, S. J. Ahn, Y. J. Cha, and S. H. Suh, "Aging Is Positively Associated with Peri-Sinus Lymphatic Space Volume: Assessment Using 3T Black-Blood MRI," J. Clin. Med. 2020, Vol. 9, Page 3353 , vol. 9, no. 10, p. 3353, Oct. 2020, doi: 10.3390/JCM9103353.

\section{Figures}




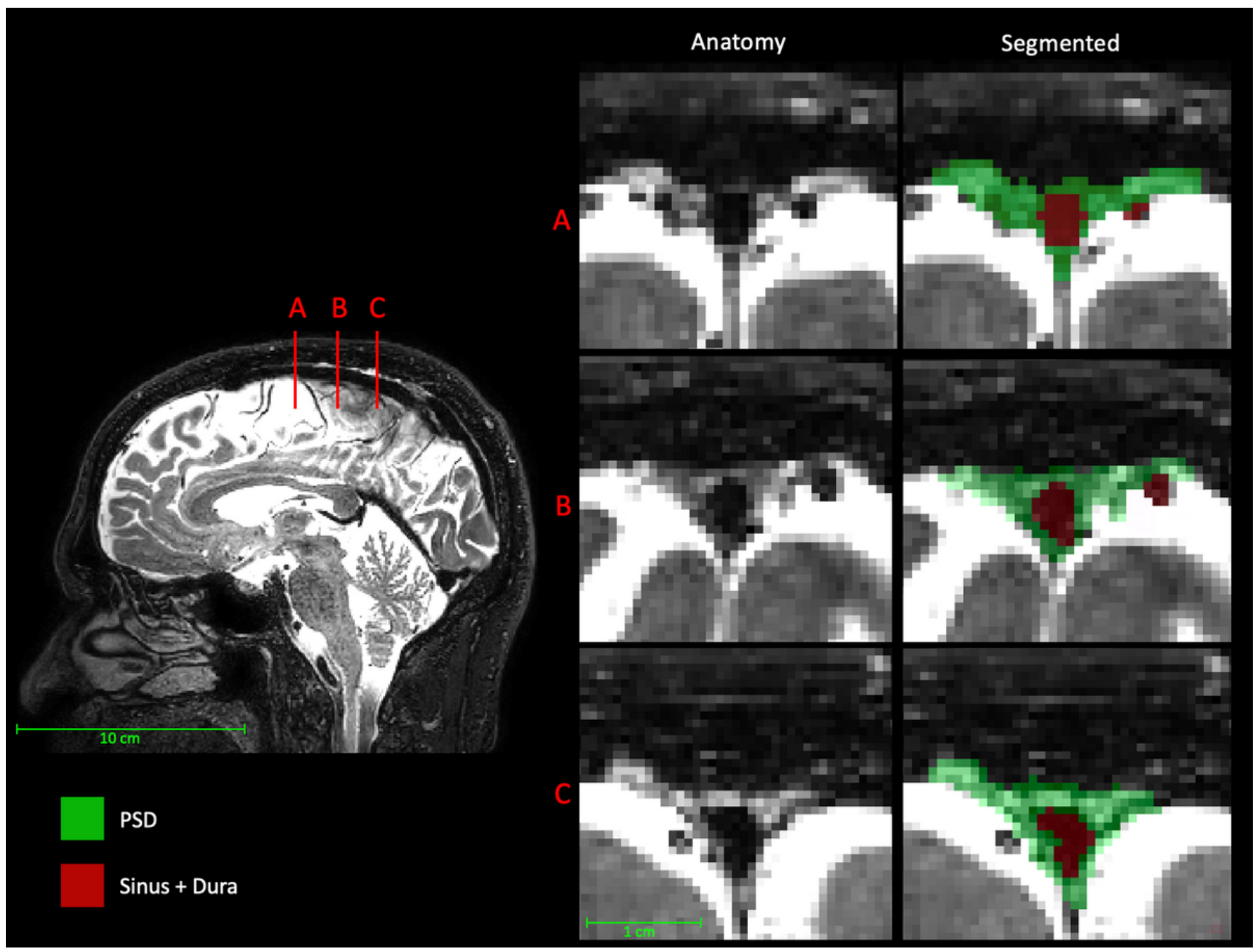

\section{Figure 1}

Parasagittal dura space (PSD) definition using T2-weighted MRI. Three coronal slices are taken from the posterior aspect of the frontal lobe to the medial aspect of the parietal lobe. In this figure the PSD appears in green, the rest of the parasagittal space appears in red (i.e., sagittal sinus, and afferent veins). 


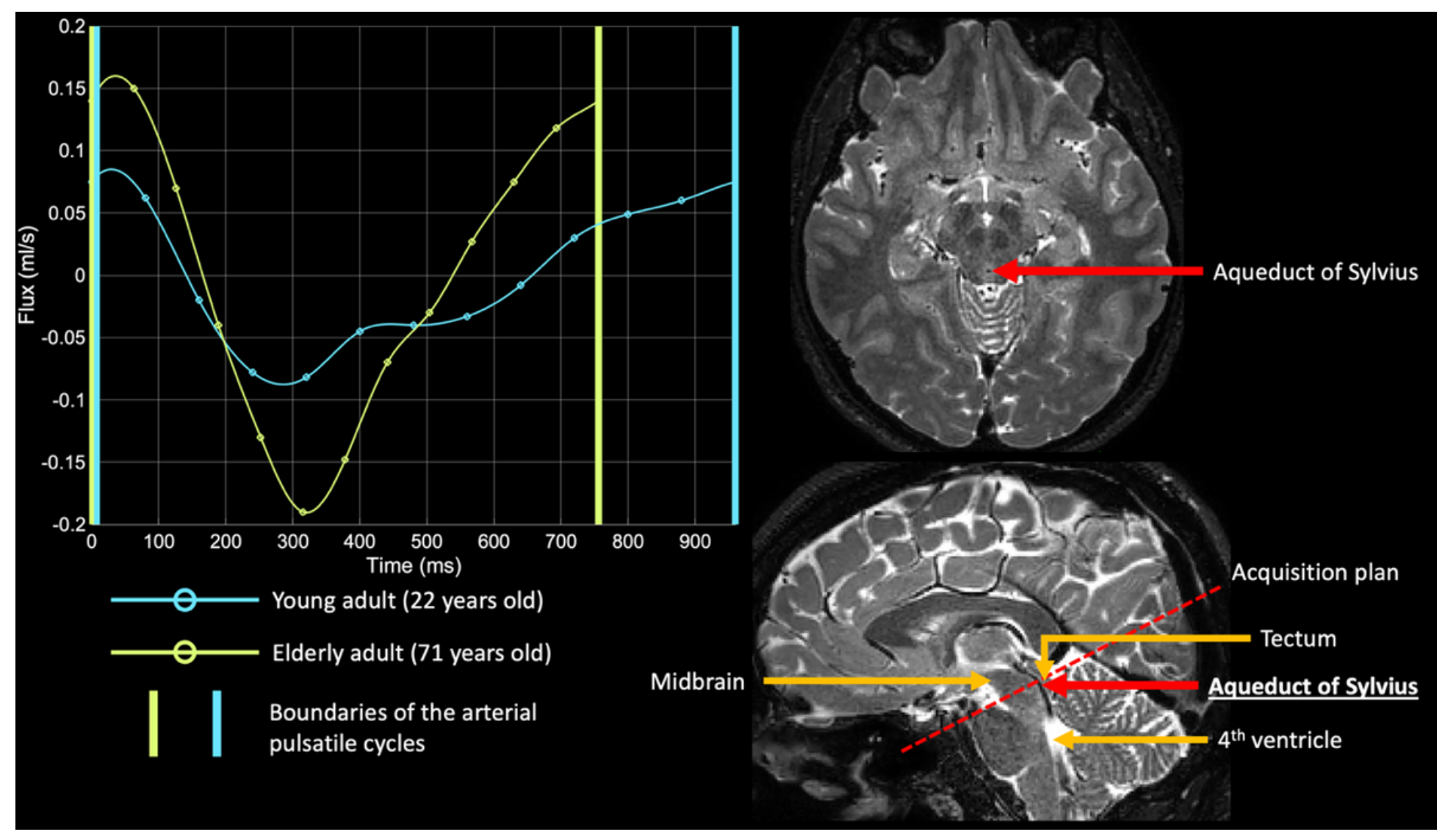

Figure 2

Illustration of cerebral aqueduct flux acquisition. On the left, two curves representing the recorded CSF flux through the aqueduct of Sylvius over one arterial pulsatile cycle for a young adult (22 years old) and an elderly subject (71 years old). On the right, oblique axial and sagittal slices of T2-weighted MRI indicating the localization of the aqueduct of Sylvius. Due to high CSF flow through the aqueduct of Sylvius, the MR signal is dephased and appears hypointense on 3D T2-weighted MRI. 

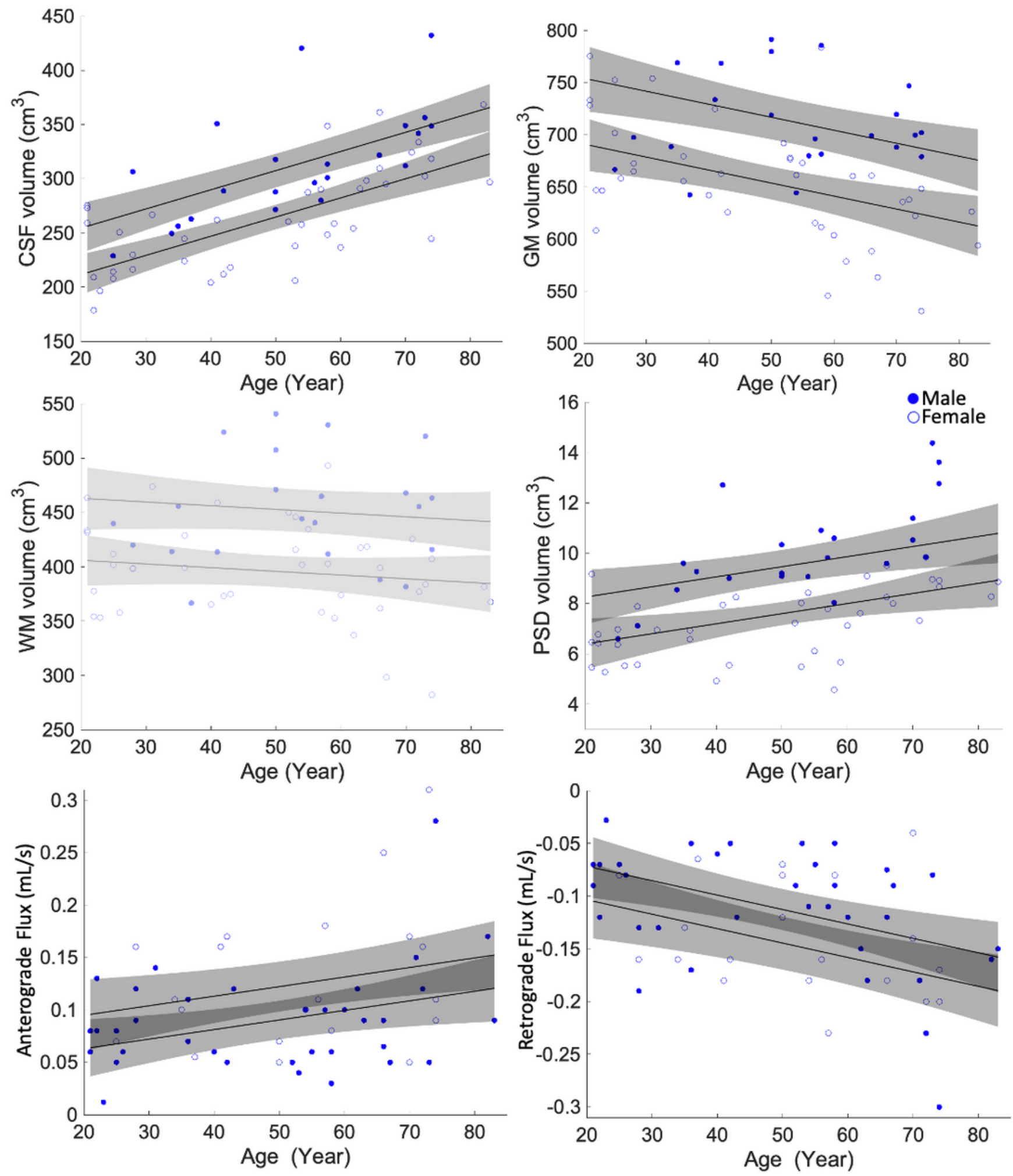

\section{Figure 3}

Relationship of age with different anatomical characteristics for cerebrospinal fluid (CSF), gray matter (GM), white matter (WM), and parasagittal dural (PSD) volumes. As expected, data show a significant correlation of GM and CSF volume with age. In addition, this study shows a novel significant relationship of normal aging with PSD volume, and CSF flux in the cerebral aqueduct. Non-significant trends appear in light gray shade; significant trends appear in dark gray shade. 

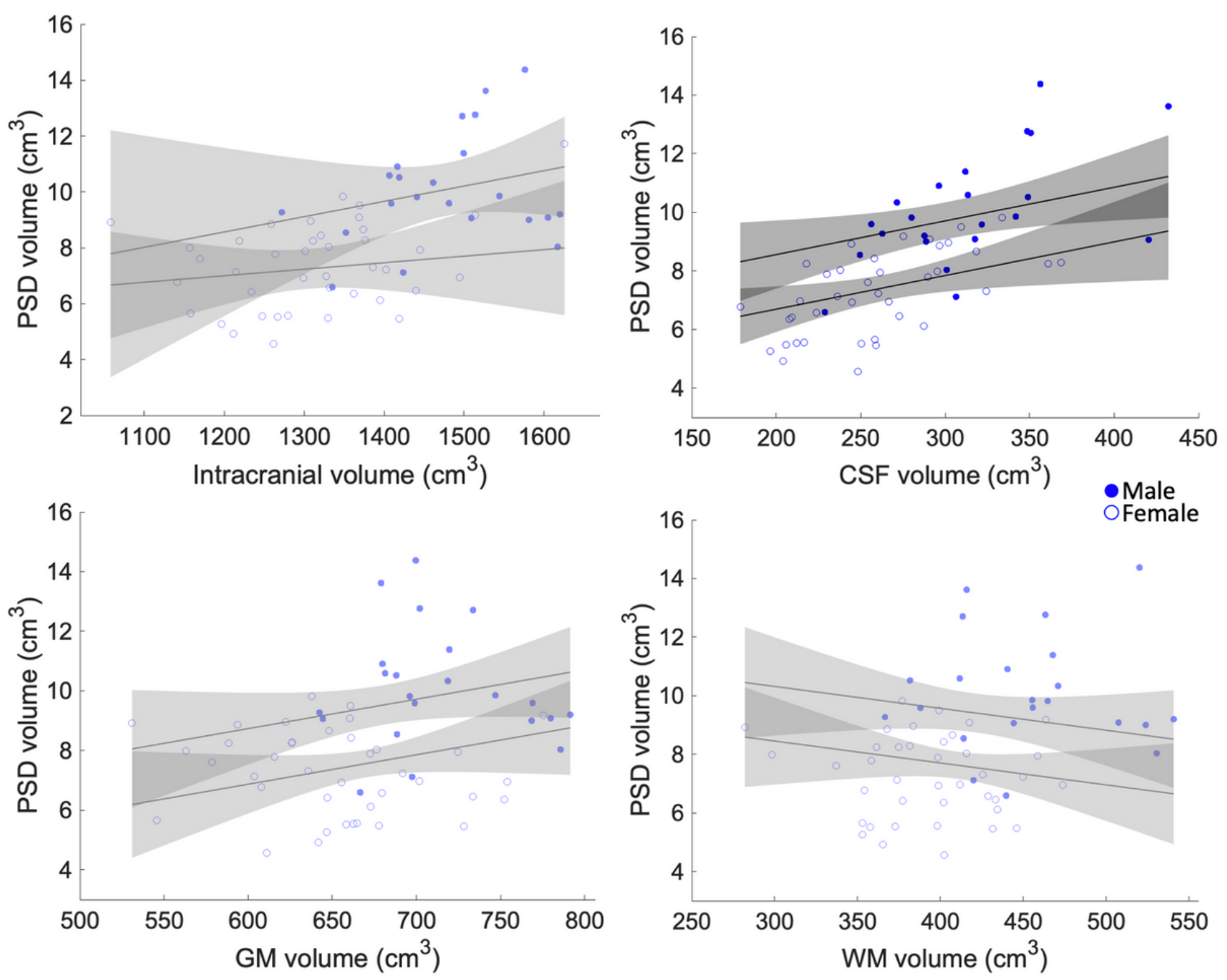

Figure 4

Correlation of parasagittal dural (PSD) and diverse anatomical measure. PSD does not show a significant relationship with total gray matter (GM) or white matter (WM). Analysis shows a significant relationship of PSD and cerebrospinal fluid (CSF) volume ( $p$-value $=0.02$, q-value $=0.04)$. Moreover, analysis shows a non-significant relationship with intracranial volume (ICV) when controlled for sex dependency. Nonsignificant trends appear in light gray shade; significant trends appear in dark gray shade. 

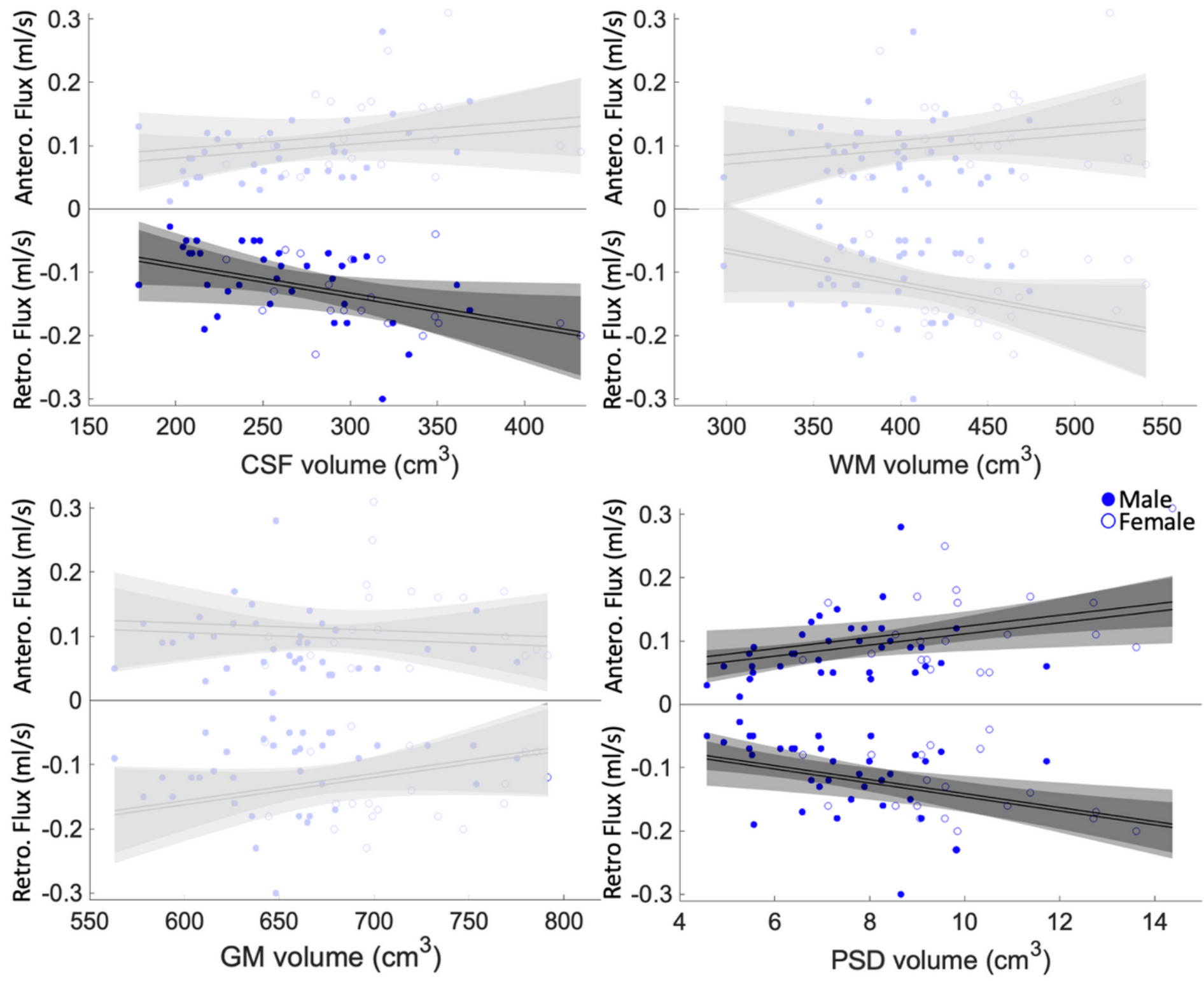

\section{Figure 5}

Analysis of relationship between anteretrograde and retrograde cerebrospinal fluid (CSF) flux in the cerebral aqueduct and CSF, gray matter (GM), white matter (WM), and parasagittal dural (PSD) volumes. The correlation analysis of WM and GM are non-significant. CSF volumes are negatively correlated with retrograde CSF flux ( $p$-value $=0.04$, q-value $=0.08$ ). PSD volume correlate with both anteretrograde and retrograde CSF flux ( $p$-values $=0.01$ and 0.001 , q-values $=0.04,0.004)$. Non-significant trends appear in light gray shade; significant trends appear in dark gray shade. 


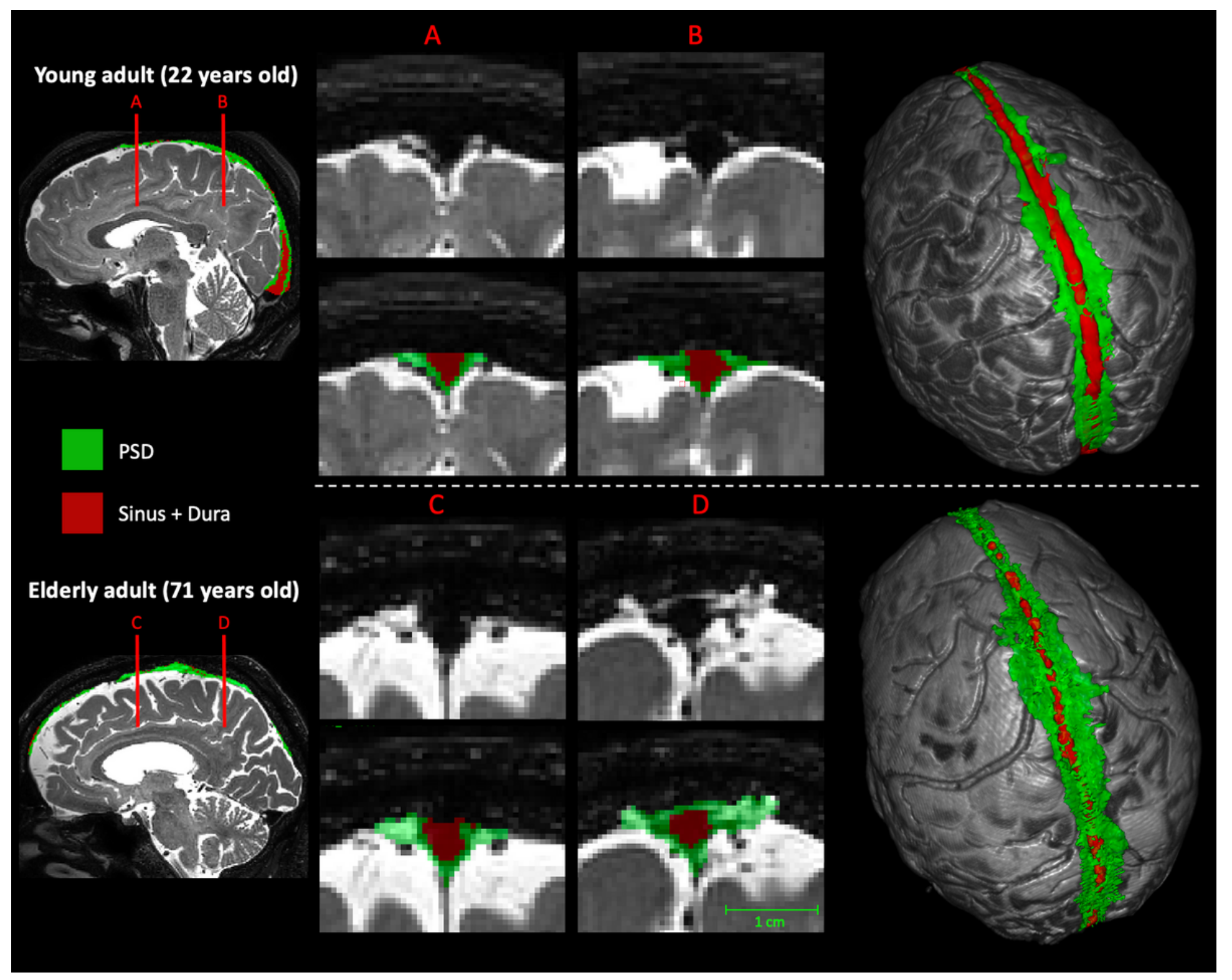

Figure 6

Difference of PSD volume between a young adult and an elderly person. Label maps are produced by the automatic segmentation method. In red, voxels assigned as sinus and contributing veins, in green voxels assigned as PSD space. The two middle columns show the slices extracted on the: $(A, C)$ posterior aspect of the frontal lobe, and (B, D) the anterior aspect of the parietal lobe (i.e., area that shows greatest PSD volume enlargement). On the right, 3D surfaces representation of the brain and its PSD.

\section{Supplementary Files}

This is a list of supplementary files associated with this preprint. Click to download.

- supplementarymaterials.docx 